\title{
EGFR Driver Mutation Negative
}

National Cancer Institute

\section{Source}

National Cancer Institute. EGFR Driver Mutation Negative. NCI Thesaurus. Code

C129575.

A genetic finding indicating that disease-causing (driver) mutations in the EGFR gene are not present in a sample. 